\title{
Low Serum Levels of EPA are Associated with the Size and Growth Rate of Abdominal Aortic Aneurysm
}

\author{
Tatsuro Aikawa ${ }^{1}$, Tetsuro Miyazaki ${ }^{1}$, Kazunori Shimada ${ }^{1}$, Yurina Sugita ${ }^{1}$, Megumi Shimizu ${ }^{1}$, Shohei Ouchi ${ }^{1}$, \\ Tomoyasu Kadoguchi ${ }^{1}$, Yasutaka Yokoyama ${ }^{2}$, Tomoyuki Shiozawa ${ }^{1}$, Masaru Hiki ${ }^{1}$, Shuhei Takahashi ${ }^{1}$, \\ Hamad Al Shahi ${ }^{1}$, Shizuyuki Dohi ${ }^{2}$, Atsushi Amano ${ }^{2}$ and Hiroyuki Daida ${ }^{1}$ \\ ${ }^{1}$ Department of Cardiovascular Medicine, Juntendo University Graduate School of Medicine, Tokyo, Japan \\ ${ }^{2}$ Department of Cardiovascular Surgery, Juntendo University Graduate School of Medicine, Tokyo, Japan
}

\begin{abstract}
Aim: Omega-3 polyunsaturated fatty acids (PUFAs) such as eicosapentaenoic acid (EPA) and docosahexaenoic acid (DHA) have been reported to reduce the risk of cardiovascular disease. However, whether omega-3 PUFAs are involved in the pathogenesis of abdominal aortic aneurysms (AAA) remains unclear.

Methods: We analyzed 67 consecutive patients admitted for the elective surgical repair of AAA. We investigated the association of serum EPA and DHA levels as well as the EPA/AA ratio with the size of AAA assessed using three-dimensional reconstructed computed tomography images.

Results: Mean patient age was $70 \pm 9$ years and 60 patients were male. Serum EPA and DHA levels were $75.2 \pm 35.7 \mu \mathrm{g} / \mathrm{mL}$ and $146.1 \pm 48.5 \mu \mathrm{g} / \mathrm{mL}$, respectively. EPA/AA ratio was $0.44 \pm 0.22$, which was lower than those in healthy Japanese subject and equivalent to those in Japanese patients with coronary artery disease as previously reported. Mean of the maximum AAA diameter was $56.4 \pm 8.9$ $\mathrm{mm}$, and serum EPA levels and EPA/AA ratio negatively correlated with it $(r=-0.32$ and $r=-0.32$, respectively). Multiple liner regression analysis showed that EPA levels were significant independent factor contributing to the maximum AAA diameter. Furthermore, low serum EPA levels and low EPA/AA ratio were significantly associated with the growth rate of AAA diameter $(r=-0.43$ and $r=-0.33$, respectively).

Conclusion: EPA levels in patients with AAA were relatively low. Low serum EPA levels and EPA/AA ratio were associated with the size and growth rate of AAA.
\end{abstract}

\section{See editorial vol. 24: 908-909}

Key words: Polyunsaturated fatty acids, Atherosclerosis, Inflammation, Coronary artery disease

This article is distributed under the terms of the latest version of CC BY-NC-SA defined by the Creative Commons Attribution License.

\section{Introduction}

Dietary intake of fish-derived omega-3 polyunsaturated fatty acids (PUFAs) such as eicosapentaenoic acid (EPA) and docosahexaenoic acid (DHA) has been recommended to reduce the risk of cardiovascular disease $^{1-4)}$. Omega-3 PUFAs have been demonstrated to

Address for correspondence: Tetsuro Miyazaki, Department of Cardiovascular Medicine, Juntendo University Graduate School of Medicine, 2-1-1 Hongo Bunkyo-ku, Tokyo 1138421, Japan

E-mail: tetsuro@juntendo.ac.jp

Received: October 12, 2016

Accepted for publication: February 1, 2017 possess anti-inflammatory, anti-fibrotic, and cardioprotective properties and improve vascular function $^{5-9)}$. Atherosclerosis and inflammation are reported to be implicated in the pathogenesis of aortic aneurysms ${ }^{10,11)}$. However, it is still unclear omega-3 PUFAs are implicated in the pathogenesis of aortic aneurysms as well as coronary artery disease (CAD). We previously reported that dietary intake of EPA and DHA prevented development of abdominal aortic aneurysms (AAAs) through the inhibition of macrophagemediated inflammation in a mouse model ${ }^{12)}$. Therefore, we assessed serum levels of omega-3 PUFAs in patients with AAA and investigated the association with size of AAA. 


\section{Methods}

\section{Study Subjects}

We enrolled 86 consecutive patients who were admitted to Juntendo University Hospital for the purpose of treatment for AAA by elective Endovascular Aneurysm Repair (EVAR) and conventional surgical repair from January 2013 to June 2016. We measured serum concentrations of PUFAs [EPA, DHA, and arachidonic acid (AA)] on admission. Eleven patients were excluded because 1 patient had an infected aneurysm, 1 patient had an endoleak after previous EVAR, 7 patients had taken pure EPA and 2 patients had received hemodialysis. Eight patients had not undergone three-dimensional (3D) reconstructed computed tomographic (CT) scan imaging before surgery. Finally, we analyzed 67 patients. We investigated whether serum omega-3 PUFAs (EPA and DHA), AA and the EPA/AA ratio are associated with the size of AAA. All subjects provided informed consent, the study protocol conforms to the ethical guidelines of the 1975 Declaration of Helsinki, and the study was approved by the ethical committee of Juntendo University Hospital.

Blood pressure (BP) was measured using a standard mercury sphygmomanometer. Height and weight were measured using an automated scale, and body mass index (BMI) was calculated as the weight in kilograms divided by the square of the height in meters. Hypertension was defined as systolic blood pressure $\geq$ $140 \mathrm{mmHg}$, diastolic blood pressure $\geq 90 \mathrm{mmHg}$, or currently taking antihypertensive medications. Dyslipidemia was defined as a low-density lipoprotein cholesterol (LDL-C) level $\geq 140 \mathrm{mg} / \mathrm{dL}$, a high-density lipoprotein cholesterol (HDL-C) level $<40 \mathrm{mg} / \mathrm{dL}$, a triglycerides (TG) level $\geq 150 \mathrm{mg} / \mathrm{dL}$, or currently taking lipid-lowering medications. Diabetes mellitus (DM) was defined as a documented history of diabetes treated with medications or hemoglobin A1c (HbA1c) of National Glycohemoglobin Standardization Program (NGSP) level $\geq 6.5 \%$, fasting plasma glucose level $\geq 126 \mathrm{mg} / \mathrm{dL}$, or non-fasting plasma glucose level $\geq 200 \mathrm{mg} / \mathrm{dL}$.

\section{Blood Sampling}

Whole blood samples were drawn after overnight fasting within $24 \mathrm{~h}$ of admission. Serum levels of total cholesterol (TC), TG, and HDL-C were measured using standard enzymatic methods, and LDL-C values were calculated using the Friedewald formula ${ }^{13)}$. Plasma glucose concentrations and $\mathrm{HbA1c}$, C-reactive protein $(\mathrm{CRP})$, and creatinine $(\mathrm{Cr})$ levels were measured using standardized methods. The estimated glomerular filtration rate was calculated based on the Jap- anese equation that uses serum $\mathrm{Cr}$ level, age, and gender as follows: estimated glomerular filtration rate $\left(\mathrm{mL} / \mathrm{min} / 1.73 \mathrm{~m}^{2}\right)=194 \times \mathrm{Cr}^{-1.094} \times$ age $^{-0.287}($ female $\times$ $0.739)^{14)}$. Serum concentrations of EPA, DHA, and AA levels were measured by SRL Inc. (Tokyo, Japan) using standard laboratory protocols.

\section{Computed Tomography, Measuring Aneurysm Diameter}

All 67 patients had undergone 3D reconstructed CT scan imaging before surgery. All CT angiographies were performed on a multidetector row CT scanner with 64 detectors (Aquilion; Toshiba, Tokyo, Japan). These CT images were reviewed on a 3D workstation (Synapse Vincent; Fujifilm, Tokyo, Japan). The aneurysm's maximum diameter was evaluated, with measurements obtained perpendicular to the centerline of aorta and aneurysm ${ }^{15,16)}$. The centerline of aorta and aneurysm was identified, and a curved multiplanar reconstruction (CPR) image was created. Then, the maximum diameter on $\mathrm{CPR}$ was determined using the cross section perpendicular to the centerline in this CPR image ${ }^{17)}$. Representative images used to determine the maximum diameter of AAA are shown in Fig. 1.

\section{Coronary Artery Disease}

CAD was defined as patients with a documented history of acute myocardial infarction, coronary artery bypass graft surgery, or documented presence of significant coronary artery stenosis (luminal narrowing $\geq$ $50 \%)$ in at least one major coronary artery by coronary angiography or multidetector computed tomographic angiography ${ }^{18,19)}$. Coronary angiography was performed according to standard methods ${ }^{20)}$. Coronary segments were analyzed according to the model of the American College of Cardiology/American Heart Association ${ }^{21}$.

\section{Statistical Analysis}

Continuous variables were expressed as mean \pm standard deviation (SD), and categorical variables were reported as percentages. Statistical differences between the groups were analyzed by unpaired Student's $t$-test, the chi-square test, Fisher's exact test, or the Mann-Whitney-Wilcoxon rank-sum test, as appropriate. Correlations between 2 variables were determined by simple linear regression analysis. Spearman correlations were used when variables were not normally distributed. Multiple linear regression analysis was used to determine the factors independently contributing to the maximum AAA diameter among the variables with a $P$ value $<0.1$ in univariate analysis (BMI, EPA and using $\beta$ blocker). All statistical 


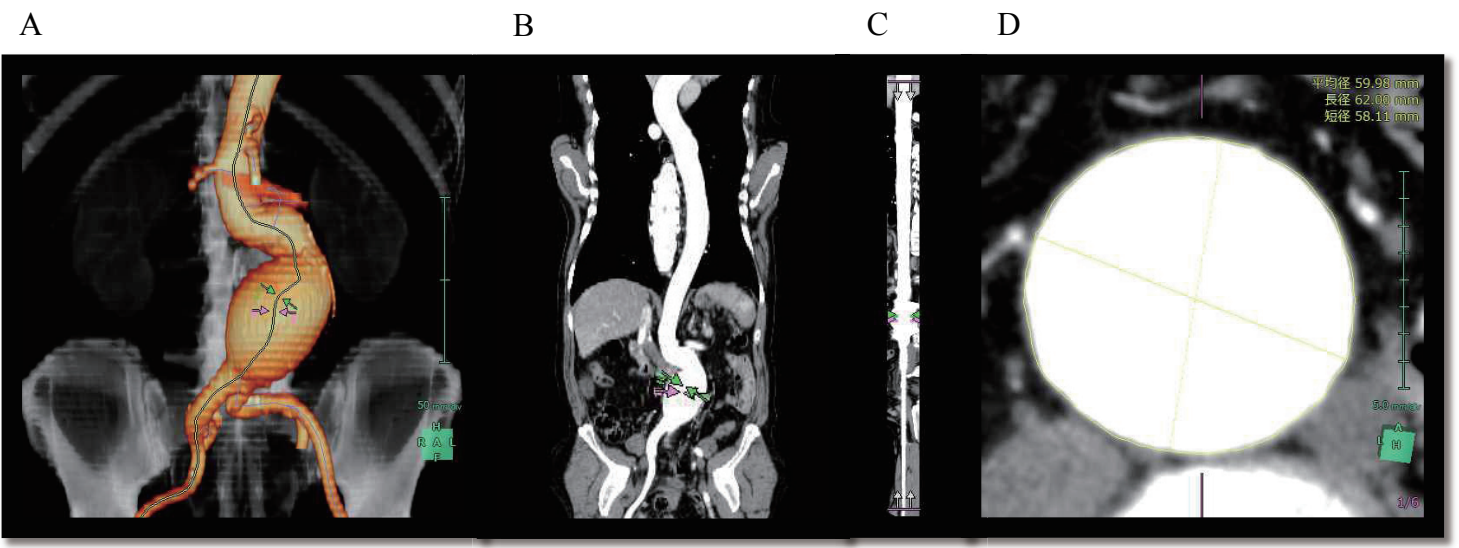

Fig. 1. Representative images to determine the maximum AAA diameter

The centerline of the aorta and aneurysm is identified and curved multiplanar reconstruction (CPR) images are created (A, B and C). Then, the maximum diameter is determined using the cross section perpendicular to the centerline in the CPR image (D).

analyses were performed using JMP 12 software for Windows (SAS Institute, Cary, NC, USA.). Statistical significance was defined as a $P$-value $<0.05$.

\section{Results}

\section{Clinical Characteristics of Patients}

The baseline characteristics of all patients are shown in Table 1. Over all 67 patients, the mean age was $70 \pm 9$ years, BMI was $24.8 \pm 2.9 \mathrm{~kg} / \mathrm{m}^{2}$, and 60 $(89.6 \%)$ were male. Nineteen $(28.4 \%)$ patients had a history of current smoking. The number of hypertension, dyslipidemia, diabetes mellitus, and coronary artery disease patients were 52 (77.6\%), 59 (88.1\%), $13(19.4 \%)$, and $40(59.7 \%)$, respectively.

\section{Serum PUFA Levels in Patients with AAA}

As shown in Table 1, serum levels of EPA, DHA, and AA were $75.2 \pm 35.7,146.1 \pm 48.5$, and $184.3 \pm$ $53.7 \mu \mathrm{g} / \mathrm{mL}$, respectively. The EPA/AA ratio was 0.44 \pm 0.22 , and the median value was 0.41 . We have previously reported serum levels of PUFAs in a healthy Japanese population living in an urban area. In subjects aged over 65 years, serum levels of EPA, DHA, and AA were $81.9 \pm 31.1,123.2 \pm 27.0$, and $119.8 \pm$ $22.7 \mu \mathrm{g} / \mathrm{mL}$, respectively. The EPA/AA ratio was 0.68 $\pm 0.22^{22)}$. Therefore, serum levels of EPA had a tendency to decrease with age, those of DHA, and AA had a tendency to increase with age, and the EPA/AA ratio was also relatively lower than in healthy Japanese subjects.

\section{Correlations between Serum PUFA Levels and AAA Diameter}

Mean of the maximum AAA diameter was 56.4 $\pm 8.9 \mathrm{~mm}$. There were no significant correlations between maximum AAA diameter and conventional risk factors (e.g., hypertension, dyslipidemia, diabetes mellitus, current smoking, and coronary artery disease). There were no significant correlations between serum levels of TC, HDL-C, LDL-C, TG, HbA1c, creatinine and CRP, and maximum AAA diameter. Although statins, $\beta$ blockers, and angiotensin pathway inhibition (angiotensin converting enzyme inhibitors or angiotensin II receptor blockers) were pharmacological treatment strategy for AAA, only $\beta$ blocker usage was associated with AAA diameter (Table 2).

As shown in Fig. 2, serum levels of EPA and the EPA/AA ratio were negatively correlated with maximum AAA diameter $(r=-0.32, P=0.0073$, and $r=-$ $0.32, P=0.0075$, respectively). However, there were no significant correlations between maximum AAA diameter and serum levels of DHA and AA. Multiple liner regression analysis showed that serum levels of EPA were significant independent factor contributing to maximum AAA diameter (EPA: $\beta=-0.269, P=$ 0.02, BMI: $\beta=0.125, P=0.2$, using $\beta$ blocker: $\beta=-$ $0.109, P=0.3)$ (Table 3).

\section{Comparison between Serum PUFA Levels between the CAD and the Non-CAD Groups}

The patients were also divided into a CAD group and a non-CAD group. Characteristics of the patients in both groups are shown in Table 1. The EPA/AA ratio was not significantly different between the two groups $(0.43 \pm 0.22$ vs. $0.45 \pm 0.23, P=0.72)$. Serum levels of EPA in the non-CAD group tended to correlate with maximum AAA diameter $(r=-0.38$, $P=0.050)$, and the EPA/AA ratio in the non-CAD group was negatively correlated with maximum AAA 
Table 1. Characteristics of the patients

\begin{tabular}{|c|c|c|c|c|}
\hline & $\begin{array}{l}\text { All patients } \\
\qquad N=67\end{array}$ & $\begin{array}{c}\text { CAD group } \\
N=40\end{array}$ & $\begin{array}{c}\text { Non-CAD group } \\
\qquad N=27\end{array}$ & $P$ value \\
\hline Age, (years) & $70 \pm 9$ & $71 \pm 9$ & $70 \pm 9$ & 0.53 \\
\hline Male, $n(\%)$ & $60(89.6)$ & $38(95.0)$ & $22(81.5)$ & 0.1 \\
\hline Body mass index $\left(\mathrm{kg} / \mathrm{m}^{2}\right)$ & $24.8 \pm 2.9$ & $24.4 \pm 2.9$ & $25.2 \pm 2.9$ & 0.26 \\
\hline Systolic blood pressure $(\mathrm{mmHg})$ & $120 \pm 13$ & $119 \pm 14$ & $123 \pm 11$ & 0.19 \\
\hline Diastolic blood pressure $(\mathrm{mmHg})$ & $69 \pm 11$ & $66 \pm 11$ & $73 \pm 11$ & 0.008 \\
\hline Current smoking, $n(\%)$ & $19(28.4)$ & $13(32.5)$ & $6(22.2)$ & 0.41 \\
\hline Hypertension, $n(\%)$ & $52(77.6)$ & $30(75)$ & $22(81.5$ & 0.76 \\
\hline Dyslipidemia, $n(\%)$ & $59(88.1)$ & $37(92.5)$ & $22(81.5)$ & 0.25 \\
\hline Diabetes mellitus, $n(\%)$ & $13(19.4)$ & $10(25.0)$ & $3(11.1)$ & 0.21 \\
\hline Coronary artery disease, $n(\%)$ & $40(59.7)$ & & & \\
\hline Total cholesterol (mg/dL) & $176 \pm 31$ & $169 \pm 26$ & $187 \pm 34$ & 0.015 \\
\hline HDL-cholesterol (mg/dL) & $45 \pm 13$ & $45 \pm 14$ & $46 \pm 11$ & 0.93 \\
\hline LDL-cholesterol (mg/dL) & $102 \pm 28$ & $96 \pm 24$ & $111 \pm 32$ & 0.028 \\
\hline Triglyceride $(\mathrm{mg} / \mathrm{dL})$ & $143 \pm 64$ & $140 \pm 61$ & $149 \pm 70$ & 0.56 \\
\hline HbA1c (NGSP) (\%) & $6.0 \pm 0.6$ & $6.1 \pm 0.7$ & $5.9 \pm 0.5$ & 0.17 \\
\hline Creatinine $(\mathrm{mg} / \mathrm{dL})$ & $0.86 \pm 0.22$ & $0.88 \pm 0.24$ & $0.82 \pm 0.19$ & 0.62 \\
\hline $\mathrm{eGFR}\left(\mathrm{mL} / \mathrm{min} / 1.73 \mathrm{~m}^{2}\right)$ & $70.1 \pm 17.6$ & $68.7 \pm 16.8$ & $72.3 \pm 18.8$ & 0.41 \\
\hline $\mathrm{CRP}(\mathrm{mg} / \mathrm{dL})$ & $0.28 \pm 0.45$ & $0.31 \pm 0.53$ & $0.24 \pm 0.32$ & 0.34 \\
\hline $\mathrm{EPA}(\mu \mathrm{g} / \mathrm{mL})$ & $75.2 \pm 35.7$ & $71.7 \pm 29.7$ & $80.5 \pm 43.2$ & 0.6 \\
\hline $\mathrm{DHA}(\mu \mathrm{g} / \mathrm{mL})$ & $146.1 \pm 48.5$ & $142.7 \pm 45.3$ & $151.0 \pm 53.4$ & 0.53 \\
\hline $\mathrm{AA}(\mu \mathrm{g} / \mathrm{mL})$ & $184.3 \pm 53.7$ & $182.8 \pm 54.9$ & $186.5 \pm 52.8$ & 0.74 \\
\hline EPA/AA ratio & $0.44 \pm 0.22$ & $0.43 \pm 0.22$ & $0.45 \pm 0.23$ & 0.72 \\
\hline Maximum AAA diameter (mm) & $56.4 \pm 8.9$ & $56.9 \pm 8.7$ & $55.6 \pm 9.2$ & 0.69 \\
\hline \multicolumn{5}{|l|}{ Medications } \\
\hline Antiplatelet, $n(\%)$ & $23(34.3)$ & $20(50.0)$ & $3(11.1)$ & 0.001 \\
\hline Calcium channel blocker, $n(\%)$ & $31(46.3)$ & $14(35.0)$ & $17(63.0)$ & 0.028 \\
\hline$\beta$-blocker, $n(\%)$ & $24(35.8)$ & $19(47.5)$ & $5(18.5)$ & 0.019 \\
\hline ACE inhibitor or ARB, $n(\%)$ & $36(53.7)$ & $23(57.5)$ & $13(48.2)$ & 0.46 \\
\hline Statin, $n(\%)$ & $46(68.7)$ & $33(82.5)$ & $13(48.2)$ & 0.006 \\
\hline
\end{tabular}

Values are presented as means \pm standard deviations. $\mathrm{HDL}=$ high-density lipoprotein, $\mathrm{LDL}=$ low-density lipoprotein, $\mathrm{HbA1c}=$ hemoglobin A1c, NGSP = national glycohemoglobin standardization program, eGFR=estimated glomerular filtration rate, $\mathrm{CRP}=\mathrm{C}$-reactive protein, $\mathrm{EPA}=$ eicosapentaenoic acid, $\mathrm{DHA}=$ docosahexaenoic acid, $\mathrm{AA}=$ arachidonic acid, $\mathrm{AAA}=\mathrm{abdominal}$ aortic aneurysm, $\mathrm{ACE}=$ angiotensin converting enzyme, $\mathrm{ARB}=$ angiotensin $\Pi$ receptor blocker.

Table 2. Univariate linear regression analysis for correlates of maximum AAA diameter

\begin{tabular}{|c|c|c|c|c|c|}
\hline & $r$ & $P$ value & & $r$ & $P$ value \\
\hline Age & -0.043 & 0.73 & HbAlc & 0.007 & 0.95 \\
\hline Gender & 0.009 & 0.94 & Creatinine & -0.054 & 0.66 \\
\hline Body mass index & 0.24 & 0.05 & eGFR & 0.091 & 0.46 \\
\hline Systolic blood pressure & -0.091 & 0.46 & CRP & -0.039 & 0.75 \\
\hline Diastolic blood pressure & -0.15 & 0.22 & EPA & -0.32 & 0.007 \\
\hline Current smoking & 0.088 & 0.47 & DHA & -0.14 & 0.25 \\
\hline Hypertension & 0.075 & 0.54 & $\mathrm{AA}$ & 0.026 & 0.83 \\
\hline Dyslipidemia & -0.11 & 0.36 & EPA/AA ratio & -0.32 & 0.008 \\
\hline Diabetes mellitus & -0.037 & 0.77 & Antiplatelet & 0.021 & 0.87 \\
\hline Coronary artery disease & 0.05 & 0.69 & Calcium channel blocker & 0.15 & 0.23 \\
\hline Total cholesterol & 0.077 & 0.53 & $\beta$-blocker & 0.21 & 0.091 \\
\hline HDL-cholesterol & -0.13 & 0.28 & ACE inhibitor or ARB & 0.015 & 0.91 \\
\hline LDL-cholesterol & 0.16 & 0.18 & Statin & -0.032 & 0.79 \\
\hline Triglyceride & 0.088 & 0.48 & & & \\
\hline
\end{tabular}

Values are presented as means \pm standard deviations. $\mathrm{CAD}=$ coronary artery disease, $\mathrm{HDL}=$ high-density lipoprotein, $\mathrm{LDL}=$ low-density lipoprotein, $\mathrm{HbA1c}=$ hemoglobin $\mathrm{A} 1 \mathrm{c}, \mathrm{NGSP}=$ national glycohemoglobin standardization program, eGFR=estimated glomerular filtration rate, $\mathrm{CRP}=\mathrm{C}$-reactive protein, $\mathrm{EPA}=$ eicosapentaenoic acid, $\mathrm{DHA}=$ docosahexaenoic acid, $\mathrm{AA}=$ arachidonic acid, $\mathrm{ACE}=$ angiotensin converting enzyme, $\mathrm{ARB}=$ angiotensin II receptor blocker. 

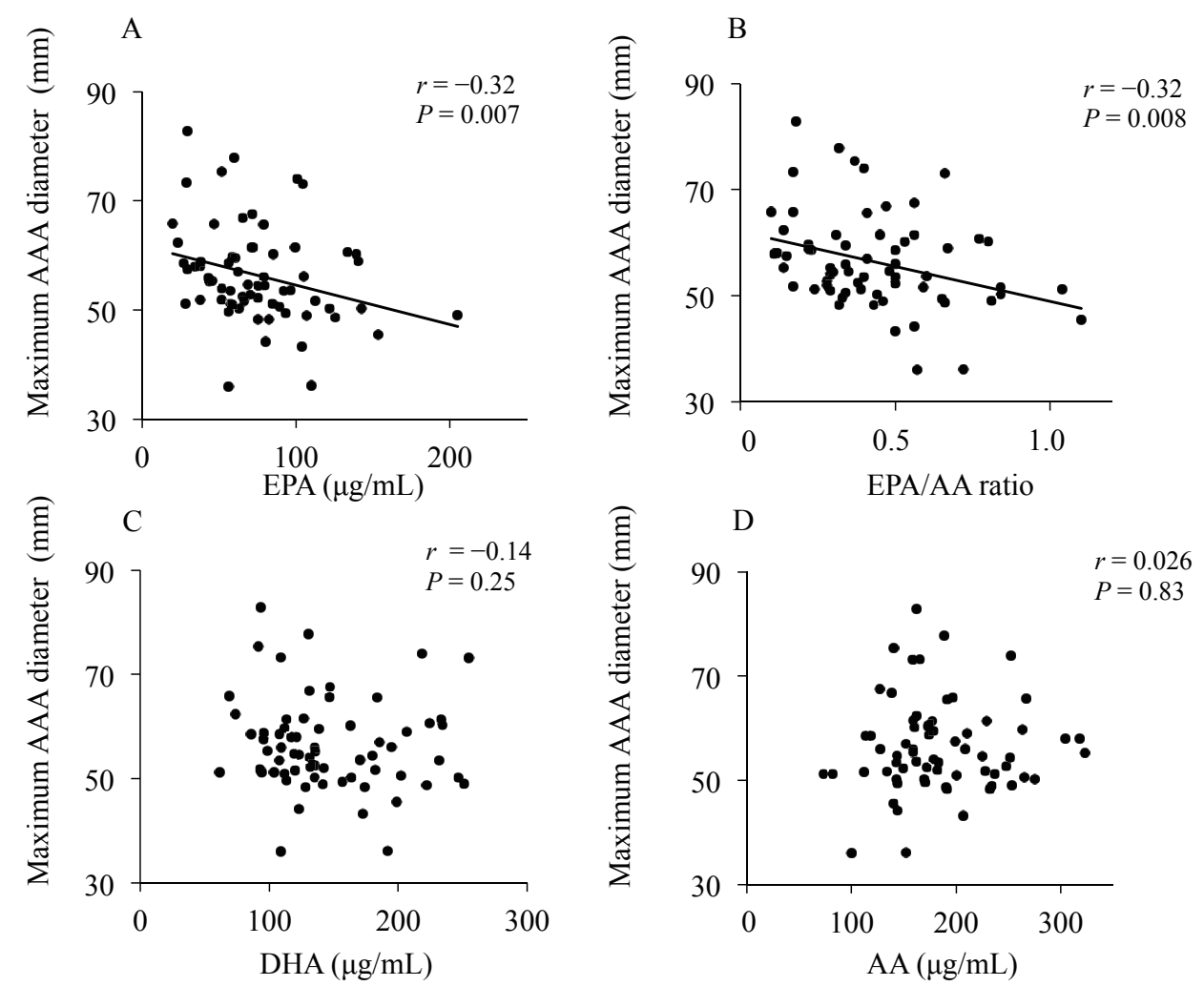

Fig. 2. Correlations between serum levels of PUFAs and maximum AAA diameter

Serum levels of EPA (A), and the EPA/AA ratio (B) were significantly negatively associated with the maximum AAA diameter but not serum levels of DHA (C) or AA (D).

AA: arachidonic acid, AAA: abdominal aortic aneurysm, DHA: docosahexaenoic acid, EPA: eicosapentaenoic acid, PUFAs: polyunsaturated fatty acids.

Table 3. Multiple liner regression analysis for correlates of maximum AAA diameter

\begin{tabular}{lrll}
\hline & $\beta$ & SE & $P$ value \\
\hline EPA & -0.269 & 0.03 & 0.028 \\
BMI & 0.125 & 0.364 & 0.29 \\
$\beta$ blocker & -0.109 & 1.1 & 0.36 \\
\hline
\end{tabular}

$\mathrm{R}^{2}=0.11, P=0.05$

Multiple linear regression analysis was performed among variables with a $P$ value $<0.1$ in univariate analysis (BMI, EPA and using $\beta$ blocker). Using $\beta$ blocker was assigned a value of 1 . Non using $\beta$ blocker was assigned a value of 0 .

diameter $(r=-0.42, P=0.030)$. Whereas maximum AAA diameter tended to be associated with both serum levels of EPA and the EPA/AA ratio $(r=-0.26$, $P=0.10$, and $r=-0.28, P=0.079$, respectively) in the CAD group (Fig. 3).

\section{Correlations between Serum PUFA Levels and Growth Rate of AAA Diameter}

We investigated the rate of AAA expansion.
Among all study subjects, for 41 patients previously performed CT scans over at least six-month intervals could be reviewed retrospectively. We evaluated aneurysms by measuring the traditional maximum minoraxis diameter using the cross sectional slice. Mean of the maximum minor-axis AAA diameter was 48.8 \pm $6.1 \mathrm{~mm}$. Mean follow-up time was $30.6 \pm 22.5$ months (range 6-77 months). The mean monthly growth rate of maximum minor-axis AAA diameter was $0.28 \pm 0.12 \mathrm{~mm} / \mathrm{month}$ (range $0.11-0.64 \mathrm{~mm}$ ). Systolic blood pressure and diastolic blood pressure were positively correlated with monthly growth rate of maximum minor-axis AAA diameter $(r=0.39$, $P=0.0097$, and $r=0.31, P=0.045$, respectively) and serum levels of creatinine was negatively correlated with monthly growth rate of maximum minor-axis AAA diameter $(r=-0.33, P=0.035)$. Serum levels of EPA and the EPA/AA ratio were negatively correlated with AAA growth rate $(r=-0.43, P=0.0056$, and $r=-0.33, P=0.033$, respectively), as shown in Fig. 4 . 

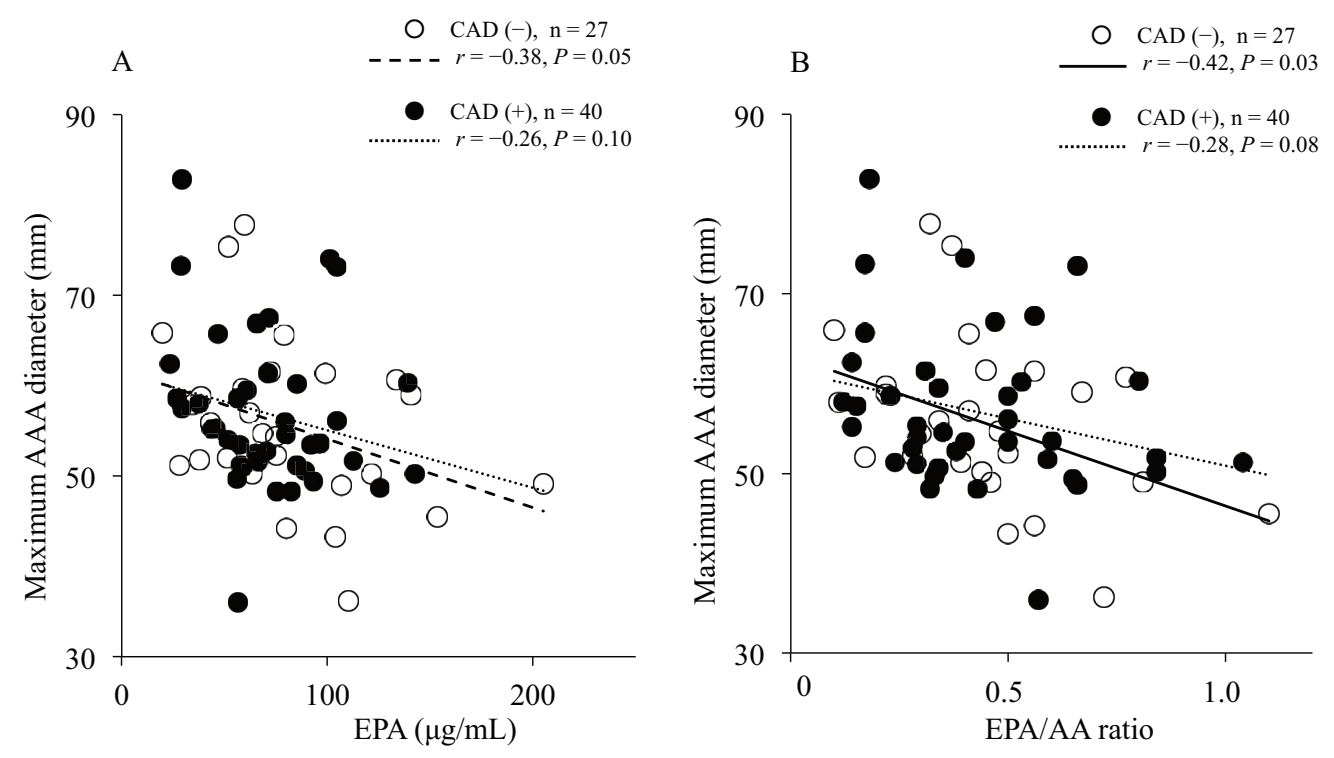

Fig.3. Correlations between serum levels of EPA and EPA/AA ratio and maximum AAA diameter in the $\mathrm{CAD}$ and the non-CAD group

Both serum levels of EPA in the non-CAD group (white circles) and the CAD group (black circles) tended to negatively correlate with maximum AAA diameter (A). The EPA/AA ratio in the non-CAD group (white circles) was significantly negatively correlated with maximum AAA diameter, whereas maximum AAA diameter tended to be negatively associated those in the CAD group (black circles) (B).

AA: arachidonic acid, AAA: abdominal aortic aneurysm, CAD: coronary artery disease, EPA: eicosapentaenoic acid.

A

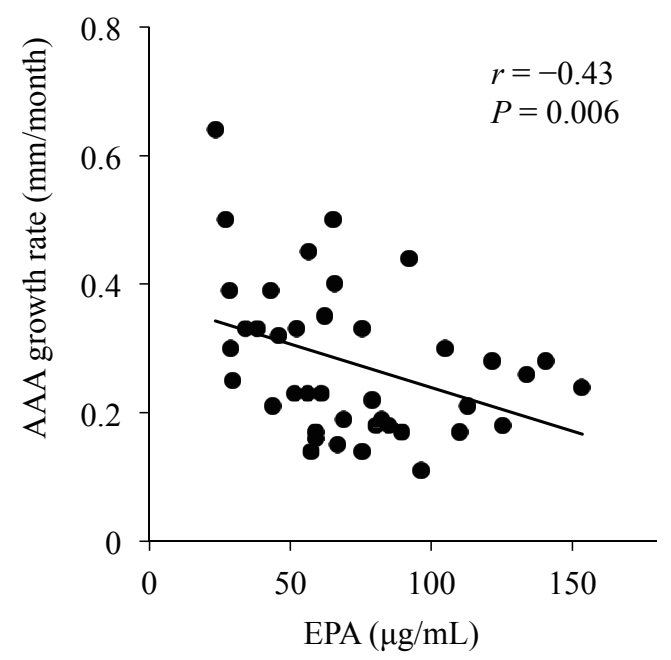

B

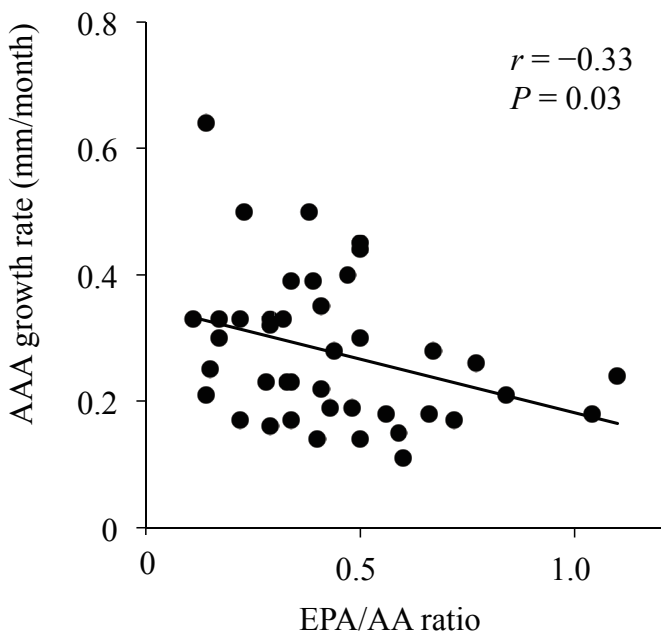

Fig. 4. Correlations between serum levels of EPA and EPA/AA ratio and growth rate of maximum minor-axis diameter of AAA

Both serum levels of EPA (A), and the EPA/AA ratio (B) were significantly negatively associated with the growth rate of maximum minor-axis diameter of AAA.

EPA: eicosapentaenoic acid, AAA: abdominal aortic aneurysm, AA: arachidonic acid. 


\section{Discussion}

To our knowledge, this is the first study to assess the association between serum levels of PUFAs and the development of AAA in a clinical setting. In patients with AAA, serum levels of EPA had a tendency to decrease with age, DHA, and AA, had a tendency to increase with age, and the EPA/AA ratio was also relatively lower than in healthy Japanese subject ${ }^{22)}$. Moreover, there were significant negative correlations between serum levels of EPA and the EPA/AA ratio and maximum AAA diameter and growth rate of AAA diameter.

Compared with our previous report of serum PUFA levels in healthy Japanese subjects ${ }^{22)}$, patients with AAA showed low EPA levels and EPA/AA ratios. Several previous studies have demonstrated that serum levels of EPA and EPA/AA ratios in patients with CAD are lower than those in normal subjects. The JELIS study showed that the average ratio of EPA/AA serum levels was 0.6 in the primary and secondary prevention study ${ }^{2}$ 23). In the Tochigi Ryoma EPA/AA Trial in Coronary Artery Disease (TREAT-CAD), the EPA/AA ratio was measured in 428 patients who underwent diagnostic coronary angiography because they were suspected to have CAD. The average EPA/ $\mathrm{AA}$ ratio was 0.49 , and its median value was $0.37^{24)}$. We also evaluated serum PUFA levels in patients with acute coronary syndrome in a metropolitan area in Japan. The EPA/AA ratio was 0.46 in 72 patients ${ }^{25)}$. The EPA/AA ratio of serum levels in our study subjects was $0.44 \pm 0.22$, which is equivalent to previous reports in patients with CAD. In addition, serum levels of EPA and the EPA/AA ratio in patients with $\mathrm{AAA}$ in the non-CAD group were equivalent to those, in the CAD group, suggesting that serum levels of $\mathrm{EPA}$, and the EPA/AA ratio in patients with AAA is relatively low regardless of the presence of CAD.

Serum levels of EPA and the EPA/AA ratio were significantly negatively correlated with maximum AAA diameter and the growth rate of AAA. AAA formation is associated with chronic aortic wall inflammation, which is linked to the production of elastinand collagen-degrading enzymes such as matrix metalloproteinases (MMP)-2 and MMP-9 ${ }^{26,27)}$. We have reported that, in the AAA model that was developed by angiotensin II infusion in apolipoprotein E-deficient mice, administration of both EPA and DHA suppressed the infiltration of macrophages and that down-regulation of inflammatory cytokines and enzymes in the aortic wall resulted in inhibition of the development of $\mathrm{AAA}^{12)}$. Wang et al. have also demonstrated that an EPA-rich diet can attenuate AAA formation in a murine $\mathrm{CaCl}_{2}$-induced $\mathrm{AAA}$ model by suppressing tissue remodeling ${ }^{28)}$. These results from in vivo studies could account for the association between lower EPA and EPA/AA ratio and the severity of AAA.

Although DHA as well as EPA has been recommended to reduce the risk of cardiovascular disease, Yagi et al. reported that the serum levels of DHA, but not EPA, are associated with the endothelial function in patients with $\mathrm{CAD}^{29)}$. Furthermore, we have reported that both EPA and DHA inhibited the development of AAA in a mouse model ${ }^{12)}$. However, serum DHA levels did not correlate with AAA formation in our study population. The mechanism(s) by which only EPA levels were associated with AAA formation is unclear. Therefore, further studies will be required to clarify the different role between DHA and EPA in the pathogenesis of AAA.

Conventional risk factors for AAA are advanced age, male gender, smoking, hypertension, dyslipidemia, and CAD. Pharmacological treatment strategies for AAA are statins, $\beta$ blockers, and angiotensin pathway inhibition. Several cohort studies have implicated that statins are associated with lower AAA growth rates $^{30,31)}$. In this study, the CAD group tended to have more conventional risk factors than the non$\mathrm{CAD}$ group, and the $\mathrm{CAD}$ group tended to use more pharmacological treatment for AAA than the nonCAD group, especially statins and $\beta$ blockers. There were stronger correlations of serum levels of EPA and EPA/AA ratio with maximum AAA diameter in the non-CAD group than in the CAD group. These results suggest that low serum levels of EPA and a low EPA/AA ratio contribute more to the development of AAA in patients with fewer conventional risk factors.

This study has several limitations. Firstly, this study was a cross-sectional survey. Therefore, we could not show the causal relationship between PUFA levels and the development of AAA. Secondly, the study sample size was relatively small. Although this study showed a significant correlation between EPA and AAA, a larger prospective study is needed to confirm our results.

\section{Conclusion}

The EPA level and EPA/AA ratio may be relatively lower in patients with AAA than in healthy Japanese subjects and equivalent to those in patients with CAD previously reported. Low levels of serum EPA and a low EPA/AA ratio are associated with the severity of AAA. Further investigation would be required to assess whether low serum levels of EPA and a low EPA/AA ratio are potential therapeutic targets. 


\section{Acknowledgements}

The authors would like to thank Enago (www. enago.jp) for the English language review.

\section{Conflict of Interest}

Dr. Daida and Dr. Shimada have received scholarship funds and lecture fees from Takeda Pharmaceutical Company Ltd. and Mochida Pharmaceutical Company Ltd. Dr. Daida has also received clinical research fundings from Takeda Pharmaceutical Company Ltd. The remaining authors report no conflicts of interest.

\section{References}

1) Teramoto T, Sasaki J, Ueshima H, Egusa G, Kinoshita M, Shimamoto K, Daida H, Biro S, Hirobe K, Funahashi T, Yokote K, Yokode M: Treatment - therapeutic lifestyle modification. J Atheroscler Thromb, 2008; 15: 109-115

2) Yokoyama $M$, Origasa $H$, Matsuzaki $M$, Matsuzawa $Y$, Saito Y, Ishikawa Y, Oikawa S, Sasaki J, Hishida H, Itakura H, Kita T, Kitabatake A, Nakaya N, Sakata T, Shimada K, Shirato K: Effects of eicosapentaenoic acid on major coronary events in hypercholesterolaemic patients (JELIS): a randomised open-label, blinded endpoint analysis. Lancet (London, England), 2007; 369: 1090-1098

3) Rizos EC, Ntzani EE, Bika E, Kostapanos MS, Elisaf MS: Association between omega-3 fatty acid supplementation and risk of major cardiovascular disease events: az systematic review and meta-analysis. JAMA, 2012; 308: 10241033

4) Ishida $T$, Naoe $S$, Nakakuki M, Kawano $H$, Imada $K$ : Eicosapentaenoic Acid Prevents Saturated Fatty AcidInduced Vascular Endothelial Dysfunction: Involvement of Long-Chain Acyl-CoA Synthetase. J Atheroscler Thromb, 2015; 22: 1172-1185

5) Schwab JM, Chiang N, Arita M, Serhan CN: Resolvin E1 and protectin D1 activate inflammation-resolution programmes. Nature, 2007; 447: 869-874

6) Bagnall RD, Weintraub RG, Ingles J, Duflou J, Yeates L, Lam L, Davis AM, Thompson T, Connell V, Wallace J, Naylor C, Crawford J, Love DR, Hallam L, White J, Lawrence C, Lynch M, Morgan N, James P, du Sart D, Puranik R, Langlois N, Vohra J, Winship I, Atherton J, McGaughran J, Skinner JR, Semsarian C: A Prospective Study of Sudden Cardiac Death among Children and Young Adults. N Engl J Med, 2016; 374: 2441-2452

7) Kris-Etherton PM, Harris WS, Appel LJ: Fish consumption, fish oil, omega-3 fatty acids, and cardiovascular disease. Circulation, 2002; 106: 2747-2757

8) Saravanan P, Davidson NC, Schmidt EB, Calder PC: Cardiovascular effects of marine omega-3 fatty acids. Lancet (London, England), 2010; 376: 540-550

9) Matsumoto M, Sata M, Fukuda D, Tanaka K, Soma M, Hirata Y, Nagai R: Orally administered eicosapentaenoic acid reduces and stabilizes atherosclerotic lesions in ApoE- deficient mice. Atherosclerosis, 2008; 197: 524-533

10) Eliason JL, Hannawa KK, Ailawadi G, Sinha I, Ford JW, Deogracias MP, Roelofs KJ, Woodrum DT, Ennis TL, Henke PK, Stanley JC, Thompson RW, Upchurch GR, Jr.: Neutrophil depletion inhibits experimental abdominal aortic aneurysm formation. Circulation, 2005; 112: 232240

11) Kajimoto K, Miyauchi K, Kasai T, Shimada K, Kojima Y, Shimada A, Niinami H, Amano A, Daida H: Short-term 20-mg atorvastatin therapy reduces key inflammatory factors including c-Jun N-terminal kinase and dendritic cells and matrix metalloproteinase expression in human abdominal aortic aneurysmal wall. Atherosclerosis, 2009; 206: 505-511

12) Yoshihara T, Shimada K, Fukao K, Sai E, SatoOkabayashi Y, Matsumori R, Shiozawa T, Alshahi H, Miyazaki T, Tada N, Daida H: Omega 3 Polyunsaturated Fatty Acids Suppress the Development of Aortic Aneurysms Through the Inhibition of Macrophage-Mediated Inflammation. Circ J, 2015; 79: 1470-1478

13) Friedewald WT, Levy RI, Fredrickson DS: Estimation of the concentration of low-density lipoprotein cholesterol in plasma, without use of the preparative ultracentrifuge. Clin Chem, 1972; 18: 499-502

14) Matsuo $S$, Imai E, Horio M, Yasuda $Y$, Tomita $K$, Nitta $K$, Yamagata K, Tomino Y, Yokoyama H, Hishida A: Revised equations for estimated GFR from serum creatinine in Japan. American journal of kidney diseases : the official journal of the National Kidney Foundation, 2009; 53: 982-992

15) Halazun KJ, Bofkin KA, Asthana S, Evans C, Henderson M, Spark JI: Hyperhomocysteinaemia is associated with the rate of abdominal aortic aneurysm expansion. Eur J Vasc Endovasc Surg, 2007; 33: 391-394; discussion 395396

16) Chaikof EL, Blankensteijn JD, Harris PL, White GH, Zarins CK, Bernhard VM, Matsumura JS, May J, Veith FJ, Fillinger MF, Rutherford RB, Kent KC: Reporting standards for endovascular aortic aneurysm repair. J Vasc Surg, 2002; 35: 1048-1060

17) Ihara $T$, Komori $K$, Yamamoto $K$, Kobayashi M, Banno $\mathrm{H}$, Kodama A: Three-dimensional workstation is useful for measuring the correct size of abdominal aortic aneurysm diameters. Ann Vasc Surg, 2013; 27: 154-161

18) Miller JM, Rochitte CE, Dewey M, Arbab-Zadeh A, Niinuma H, Gottlieb I, Paul N, Clouse ME, Shapiro EP, Hoe J, Lardo AC, Bush DE, de Roos A, Cox C, Brinker J, Lima JA: Diagnostic performance of coronary angiography by 64-row CT. N Engl J Med, 2008; 359: 2324-2336

19) Budoff MJ, Dowe D, Jollis JG, Gitter M, Sutherland J, Halamert E, Scherer M, Bellinger R, Martin A, Benton R, Delago A, Min JK: Diagnostic performance of 64-multidetector row coronary computed tomographic angiography for evaluation of coronary artery stenosis in individuals without known coronary artery disease: results from the prospective multicenter ACCURACY (Assessment by Coronary Computed Tomographic Angiography of Individuals Undergoing Invasive Coronary Angiography) trial. J Am Coll Cardiol, 2008; 52: 1724-1732

20) Dohi T, Miyauchi K, Ohkawa R, Nakamura K, Kurano M, Kishimoto T, Yanagisawa N, Ogita M, Miyazaki T, 
Nishino A, Yaginuma K, Tamura H, Kojima T, Yokoyama K, Kurata T, Shimada K, Daida H, Yatomi Y: Increased lysophosphatidic acid levels in culprit coronary arteries of patients with acute coronary syndrome. Atherosclerosis, 2013; 229: 192-197

21) Austen WG, Edwards JE, Frye RL, Gensini GG, Gott VL, Griffith LS, McGoon DC, Murphy ML, Roe BB: A reporting system on patients evaluated for coronary artery disease. Report of the Ad Hoc Committee for Grading of Coronary Artery Disease, Council on Cardiovascular Surgery, American Heart Association. Circulation, 1975; 51: 5-40

22) Yanagisawa N, Shimada K, Miyazaki T, Kume A, Kitamura Y, Ichikawa R, Ohmura H, Kiyanagi T, Hiki M, Fukao K, Sumiyoshi K, Hirose K, Matsumori R, Takizawa H, Fujii K, Mokuno H, Inoue N, Daida H: Polyunsaturated fatty acid levels of serum and red blood cells in apparently healthy Japanese subjects living in an urban area. J Atheroscler Thromb, 2010; 17: 285-294

23) Itakura H, Yokoyama M, Matsuzaki M, Saito Y, Origasa H, Ishikawa Y, Oikawa S, Sasaki J, Hishida H, Kita T, Kitabatake A, Nakaya N, Sakata T, Shimada K, Shirato K, Matsuzawa Y: Relationships between plasma fatty acid composition and coronary artery disease. J Atheroscler Thromb, 2011; 18: 99-107

24) Kitagawa Y, Abe S, Toyoda S, Watanabe S, Ebisawa K, Murakami Y, Takahashi T, Sugimura H, Taguchi I, Inoue T: Gender differences in the ratio of eicosapentaenoic acid to arachidonic acid in an inland prefecture, Tochigi: Tochigi Ryomo EPA/AA Trial in Coronary Artery Disease (TREAT-CAD). Internal medicine (Tokyo, Japan), 2014; 53: $177-182$

25) Nishizaki Y, Shimada K, Tani S, Ogawa T, Ando J, Taka- hashi M, Yamamoto M, Shinozaki T, Miyauchi K, Nagao K, Hirayama A, Yoshimura M, Komuro I, Nagai R, Daida $\mathrm{H}$ : Significance of imbalance in the ratio of serum $n-3$ to n-6 polyunsaturated fatty acids in patients with acute coronary syndrome. Am J Cardiol, 2014; 113: 441-445

26) Longo GM, Xiong W, Greiner TC, Zhao Y, Fiotti N, Baxter BT: Matrix metalloproteinases 2 and 9 work in concert to produce aortic aneurysms. The Journal of clinical investigation, 2002; 110: 625-632

27) Freestone T, Turner RJ, Coady A, Higman DJ, Greenhalgh RM, Powell JT: Inflammation and matrix metalloproteinases in the enlarging abdominal aortic aneurysm. Arterioscler Thromb Vasc Biol, 1995; 15: 1145-1151

28) Wang JH, Eguchi K, Matsumoto S, Fuji K, Komuro I, Nagai R, Manade I: The $\omega-3$ Polyunsaturated fatty acid, eicosapentaenoic acid, attenuates abdominal aortic aneurysm development via suppression of tissue remodeling. PLoS One, 2014; 9: e96286

29) Yagi S, Aihara K, Fukuda D, Takashima A, Hara T, Hotchi J, Ise T, Yamaguchi K, Tobiume T, Iwase T, Yamada H, Soeki T, Wakatsuki T, Shimabukuro M, Akaike M, Sata M: Effects of docosahexaenoic Acid on the endothelial function in patients with coronary artery disease. J Atheroscler Thromb, 2015; 22: 447-454

30) Moll FL, Powell JT, Fraedrich G, Verzini F, Haulon S, Waltham M, van Herwaarden JA, Holt PJ, van Keulen JW, Rantner B, Schlosser FJ, Setacci F, Ricco JB: Management of abdominal aortic aneurysms clinical practice guidelines of the European society for vascular surgery. Eur J Vasc Endovasc Surg, 2011; 41 Suppl 1: S1-s58

31) Golledge J, Norman PE: Current status of medical management for abdominal aortic aneurysm. Atherosclerosis, 2011; 217: 57-63 